\title{
ON THE ORDER OF THE AUTOMORPHISM GROUP OF A FINITE GROUP
}

W. R. SCOTT

Let $G$ be a finite group, $A(G)$ its group of automorphisms, and $p$ a prime. Several authors have given upper bounds on the order $o(A(G))$ of $A(G)$ in terms of $o(G)$. Birkhoff and Hall [1, p. 499] suggested the problem of determining a lower bound for $o((A) G)$ in terms of $o(G)$. For Abelian groups, this had already been done by Hilton [3]. He proved that if $p^{n} \mid o(G)$, then $p^{n-1}(p-1) \mid o(A(G))$ for Abelian groups $G$. The only result obtained in the general case is due to Herstein and Adney [2]. They proved that if $p^{2} \mid o(G)$, then $p \mid o(A(G))$.

In this note, it is shown that if $p^{3} \mid o(G)$, then $p^{2} \mid o(A(G))$. One is tempted to conjecture that there is a function $f(n)$ such that if $p^{f(n)} \mid o(G)$, then $p^{n} \mid o(A(G))$. However the methods used here will not suffice to prove even the case $n=3$.

Let $I(G)$ be the group of inner automorphisms of $G$. Let the operation in $G$ be addition. The union of subgroups $H$ and $K$ will be denoted by $H \cup K$, the direct sum by $H+K$, the factor group by $H / K$, and the point set difference by $H-K$. Let $[H: K]$ denote the index of $K$ in $H$.

Lemma 1. If $G$ is Abelian and $p^{n} \mid o(G)$, then $p^{n-1} \mid o(A(G))$.

This was proved in [3].

Lemma 2. Let $G=S \cup M$ where $S$ is a subgroup and $M$ a normal subgroup of $G$, and where $S \cap M=0$. Let $Z(G)$ be the center of $G$. Let $\alpha^{\prime}$ be an automorphism of $S$ such that $s \alpha^{\prime}-s \in Z(G)$ for all $s \in S$. Let $(s+m) \alpha=\left(s \alpha^{\prime}\right)+m$ for $s \in S, m \in M$. Then $\alpha$ is an automorphism of $G$.

LeMma $3 .{ }^{1}$ If $S$ is an Abelian group of order $p^{n}, n>1$, and $Z$ is a subgroup of index $p$, then there is a group $U \subseteq A(S)$ with $o(U)=p^{n-2}$ such that $s \alpha-s \in Z$ for all $s \in S$ and all $\alpha \in U$.

Proof. Let $w$ be an element of smallest order in $S-Z$. If $p^{r} w=p^{r} z$, then $p^{r}(w-z)=0$. It follows that $\{p w\}$ is pure in $Z$. Hence $Z=\{p w\}$ $+T$ and $S=\{w\}+T, T \subseteq Z$. Let $o(w)=p^{i}, o(T)=p^{i}$. Let $V^{\prime}$ be the group of automorphisms $\alpha$ of $\{w\}$ such that $w \alpha=(1+\lambda p) w$. Then

Received by the editors June 14, 1953.

${ }^{1}$ Lemma 3 is due to the referee. It permitted the proof of the present Theorem 2, which was the only theorem proved in the original version of the paper, to be used as a proof of Theorem 1 with only minor changes. 
$o\left(V^{\prime}\right)=p^{i-1}$. Let $W^{\prime}$ be a subgroup of $A(T)$ of order $p^{j-1}$ whose existence is guaranteed by Lemma 1 . There are subgroups $V$ and $W$ of $A(S)$ corresponding in an obvious way to $V^{\prime}$ and $W^{\prime}$. The group $U=\{V, W\}$ has the required properties (if $\{p w\}$ or $T$ is 0 , then $V$ or $W$, respectively, is defined to be the identity).

Theorem 1. Let $G$ be a finite group, $S$ a Sylow p-subgroup of order $p^{n}$, and $Z$ the intersection of $S$ with the center of $G$. If $[S: Z] \geqq p^{n-1}$, $[S: Z]=1$, or $[S: Z]=p$, then $p^{n-1} \mid o(A(G))$.

Proof. Case 1. $[S: Z] \geqq p^{n-1}$. Then $p^{n-1}|o(I(G))| o(A(G))$.

Case 2. $[S: Z]=1$. Let $\sigma$ be the transfer of $G$ into $S$. If $z \in Z$, then $z \sigma=[G: S] z$, hence $Z \sigma=Z$. Therefore, if $N$ is the kernel of $\sigma, G=Z+N$. By Lemma 1, $p^{n-1}|o(A(Z))| o(A(G))$.

Case 3. $[S: Z]=p$. Since the center $Z(S)$ of $S$ contains $Z$, and since $S / Z(S)$ is not cyclic of order $p, S$ is Abelian. Let $\sigma$ be the transfer of $G$ into $S$. Then $Z \sigma=Z$. If $G \sigma=Z$, then, as in case $2, G=Z+N$. By Lemma 1, there is a group $U^{\prime} \subseteq A(Z)$ with $o\left(U^{\prime}\right)=p^{n-2}$. Let $U$ be the corresponding subgroup of $A(G)$. Since $[S: Z]=p$, an element $s_{0}$ $\in S-Z$ induces an automorphism $\rho$ of order $p$. If $\alpha \in U$, then $\alpha \rho=\rho \alpha$, and $\rho \notin U$. Therefore $\{\rho, U\}$ is a subgroup of $A(G)$ of order $p^{n-1}$.

Next, assume that $G \sigma=S$. If $M$ is the kernel of $\sigma$, then $o(M)$ $=[G: S]$, and therefore $S \cap M=0, S \cup M=G$, and $M$ is normal in $G$. By Lemma 3, there is a group $U^{\prime}$ of automorphisms of $S$ with $o\left(U^{\prime}\right)$ $=p^{n-2}$ such that $s \alpha^{\prime}-s \in Z$ for all $s \in S$ and all $\alpha^{\prime} \in U^{\prime}$. By Lemma 2, there is a corresponding group $U$ of automorphisms of $G$. Let $\rho$ be defined as before, let $\alpha^{\prime} \in U^{\prime}$, and let $\alpha$ be the corresponding element of $U$ (see Lemma 2). Then

$$
(s+m) \alpha \rho=s \alpha^{\prime}+m \rho=(s+m) \rho \alpha .
$$

Since $\rho$ induces the identity automorphism on $S, \rho \notin U$, and $\{\rho, U\}$ is a subgroup of $A(G)$ of order $p^{n-1}$.

TheOREM 2. If $p^{3} \mid o(G)$, then $p^{2} \mid o(A(G))$.

PROOF. This follows immediately from Theorem 1, and the remark made in Case 1 of its proof.

\section{BIBLIOGRAPHY}

1. G. Birkhoff and P. Hall, On the order of groups of automorphisms, Trans. Amer. Math. Soc. vol. 39 (1936) pp. 496-499.

2. I. N. Herstein and J. E. Adney, A note on the automorphism group of a finite group, Amer. Math. Monthly vol. 59 (1952) pp. 309-310.

3. H. Hilton, On the order of the group of automorphisms of an Abelian group, Messenger of Mathematics (2) vol. 38 (1909) pp. 132-134. 